\title{
二硼化镁的热氧化特性研究
}

\author{
郭 洋 ${ }^{1,2}$, 张 炜 $^{2}$, 周 星 ${ }^{2}$, 邓 蕾 ${ }^{2}$ \\ (1. 军事科学院, 北京 $100091 ; 2$. 国防科技大学 空天科学学院, 长沙 410073)
}

\begin{abstract}
摘 要: 利用氧弹式量热计、热分析仪器、高温管式炉等实验装置及手段, 研究了 $\mathrm{MgB}_{2}$ 颗粒的热氧化特性和能量 释放特性。结果表明, $\mathrm{MgB}_{2}$ 的实测燃烧热值和燃烧效率均高于无定型硼。在 298 1673 K 的温度区间内, $\mathrm{MgB}_{2}$ 在缓 慢升温条件下的热氧化反应包含四个阶段, 其主要的氧化放热和增重都发生在 1200 1665 K 之间。而无定型嗍主要 的氧化放热和增重都发生在 $1919 \mathrm{~K}$ 附近。在 $1665 \mathrm{~K}$ 时, $\mathrm{MgB}_{2}$ 的氧化率高达 $94.3 \%$, 而无定型硼的氧化率仅为 $43.6 \%$ 。与无定形硼相比, $\mathrm{MgB}_{2}$ 可以在更低温度下充分氧化, 热氧化特性优于无定形嗍。
\end{abstract}

关 键 词: 嗍; 二硼化镁; 氧化特性; 燃烧效率

中图分类号: TJ55 文献标识码: A

\section{Oxidation Characteristics of Magnesium Diboride}

\author{
GUO Yang ${ }^{1,2}$, ZHANG Wei $^{2}$, ZHOU Xing $^{2}$, DENG Lei ${ }^{2}$
}

(1. PLA Academy of Military Science, Beijing 100091, China; 2. College of Aerospace Science and Engineering, National University of Defense Technology, Changsha 410073, China)

\begin{abstract}
The oxidation characteristics and energy releasing characteristics of $\mathrm{MgB}_{2}$ were studied using oxygen bomb calorimeter, thermal analyzer and tube furnace. Results show that the combustion heat and combustion efficiencies of $\mathrm{MgB}_{2}$ are all higher than those of amorphous boron. In the temperature range explored (298-1673 K), four successive phases are observed in the oxidation process of $\mathrm{MgB}_{2}$ under slow heating rate. The primary oxidation stage of $\mathrm{MgB}_{2}$ occurrs between $1200 \mathrm{~K}$ and $1665 \mathrm{~K}$. However, the primary oxidation stage of amorphous boron occurrs nearly $1919 \mathrm{~K}$. At $1665 \mathrm{~K}$, the oxidation percentage of $\mathrm{MgB}_{2}$ is $94.3 \%$, compared to $43.6 \%$ of amorphous boron. Compared with amorphous boron, the fact that complete oxidation of $\mathrm{MgB}_{2}$ can be achieved at lower temperature indicates its superiority.
\end{abstract}

Key words: boron; magnesium diboride; oxidation characteristics; combustion efficiency

硼具有极高的质量热值和体积热值，是最具前 景的高能金属燃料, 并广泛应用于含硼富燃料推进 剂。但单质嗍的熔点和沸点较高, 导致其点火和燃 烧性能较差, 进而影响其化学潜能的发挥。为解决 该难题, 现阶段主要有以下技术方案: (1)在含硼推 进剂制备过程中添加镁、铝等易燃金属 ${ }^{[1-3]}$; (2)采用 氟化物 ${ }^{[4]} 、 \operatorname{AP}(\text { 高氯酸铵 })^{[5-6]}, \operatorname{PGAP}($ 聚叠氮缩水甘
油醚 $)^{[7]}$ 及镁 ${ }^{[8-9]}$ 等物质包覆硼颗粒表面，改善其点 火和持续然烧性能; (3)对嶰颗粒进行团聚造粒 ${ }^{[10-13]}$, 改善推进剂的工艺性能, 并提高推进剂的燃烧性能; (4)在推进剂配方中采用含能粘合剂 ${ }^{[7,14-15]}$, 改善含 嗍富燃料推进剂的燃烧性能。虽然上述方法可改变 硼颗粒燃烧时的外部环境, 并部分改善其点火和持 续燃烧性能，但它们并未改变单质嗍自身的燃烧特

收稿日期：2018-09-14; 收到修改稿日期：2019-02-02

基金项目：武器装备预研基金项目(9140A28020312KG01082)

Weapons and Equipment Advanced Research Program (9140A28020312KG01082)

作者简介: 郭 洋(1984-), 男, 助理研究员. E-mail: guoyangnudt@gmail.com

通讯作者：张 炜，教授. E-mail: wzhang_nudt@nudt.edu.cn; 周 星，讲师. E-mail: xingzhou_nudt@nudt.edu.cn 
性, 硼颗粒的燃烧效率仍有待提升。

本研究提出采用二㸴化镁替代单质嗍来制备富 燃料推进剂，以期提升含嗍推进剂的燃烧效率。 $\mathrm{Hsia}^{[16]}$ 、Mestwerdt 等 ${ }^{[17-18]}$ 、Mota 等 ${ }^{[19-21]}$ 分别研究 了镁嗍化合物、锂嗍化合物及铝硼化合物的点火和 燃烧性能。研究表明, 上述硼化合物具有燃烧速度 快、燃烧效率高的优点。但上述研究侧重于嗍化合 物性能的表征, 而对于其在能量释放过程中发生的 反应及其相关机理阐述不足。鉴于此, 本研究选取 $\mathrm{MgB}_{2}$ 作为研究对象, 对其氧化反应历程进行深入 研究, 并与无定型硣的热氧化特性进行对比, 综合 分析 $\mathrm{MgB}_{2}$ 在富燃料推进剂中的应用前景。

\section{1 实验方法}

\section{1 燃烧热测试实验}

采用 GR3500 型氧弹式量热计, 测定 $\mathrm{MgB}_{2}$ (纯 度: 93.6\%)及无定型硼(纯度: $93.0 \%$ )的燃烧热, 样品 用量约为 $40 \mathrm{mg}$, 充氧气压强为 $2.6 \mathrm{MPa}$ 。

\section{2 热分析及管式炉实验}

无定型硼和 $\mathrm{MgB}_{2}$ 的热分析实验采用 STA449C 热重一差热分析 (TG-DTA) 仪进行, 空气流量为 $40 \mathrm{~mL} \cdot \mathrm{min}^{-1}$, 样品质量为 $10 \sim 20 \mathrm{mg}$, 加热速率 $5 \mathrm{~K} \cdot \mathrm{min}^{-1}$, 加热范围为室温至 $1665 \mathrm{~K}$ 。

由于热分析仪器所用样品量较少, 不便于对热 氧化产物进行后续检测分析, 故采用高温管式炉模 拟热分析实验, 以批量获取 $\mathrm{MgB}_{2}$ 在不同热氧化阶 段的氧化产物。向管式炉中通入空气流, 以相同的 升温速率将装有粉状 $\mathrm{MgB}_{2}$ 样品的刚玉坩埚加热至 目标温度, 然后停止加热, 样品随炉自然冷却, 并 分别记录样品氧化前后的质量。

\section{3 氧化产物分析实验}

采用 $\mathrm{X}$ 射线衍射(XRD)仪、扫描电子显微镜 $(\mathrm{SEM}) 、 X$ 射线能谱(EDS)仪等仪器对 $\mathrm{MgB}_{2}$ 的热氧 化产物进行检测。同时, 参考嗍镁矿石的成分分析

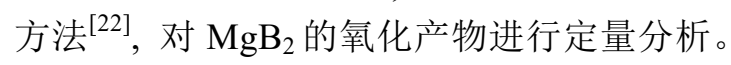

\section{2 结果与讨论}

\section{1 无定型嗍和 $\mathrm{MgB}_{2}$ 的能量释放特性}

无定型嗍和 $\mathrm{MgB}_{2}$ 的燃烧热及燃烧效率如表 1 所示。在纯氧环境中, $\mathrm{MgB}_{2}$ 的燃烧热值略高于无定 型硣，但燃烧效率(62\%)却明显高于无定型硼(38\%), 具备良好的应用基础。

\section{$2.2 \mathrm{MgB}_{2}$ 的热氧化特性}

如图 1 所示, 根据热重曲线的变化和 DTA 曲线
表 1 无定型硼和 $\mathrm{MgB}_{2}$ 的燃烧热及燃烧效率

Table 1 Combustion heats and combustion efficiencies of amorphous boron and $\mathrm{MgB}_{2}$

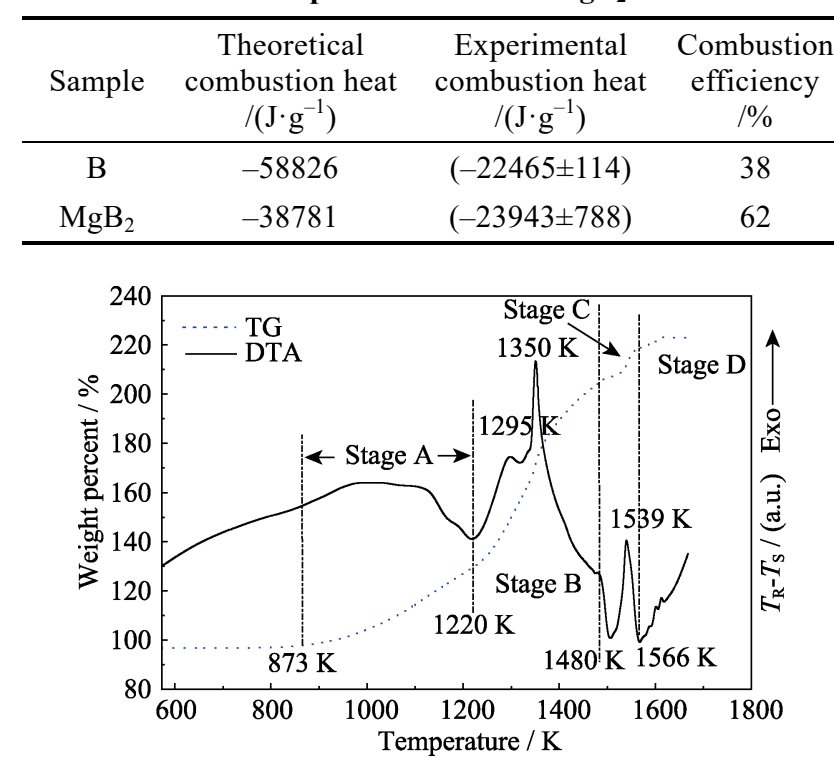

图 1 空气气氛中 $\mathrm{MgB}_{2}$ 的热氧化 $\mathrm{TG}$ 和 DTA 曲线

Fig. 1 TG and DTA curves of $\mathrm{MgB}_{2}$ oxidation in air flow

中的放热峰位置可将 $\mathrm{MgB}_{2}$ 的热氧化曲线分割为四 段进行分析。(1) 873 1220 K 为第一段, 增重量为 $29.4 \%$, 并伴有放热峰; (2) 1220 1480 K 为第二段, 增重量为 $74.8 \%$ 。 $\mathrm{MgB}_{2}$ 的氧化增重主要发生在该阶 段, 并在 $1295 、 1350 \mathrm{~K}$ 处伴有较强的放热峰; (3) 1480 1566 K 为第三段, 增重量为 $14.4 \%$, 并在 $1539 \mathrm{~K}$ 处伴有放热峰; (4) $1566 \mathrm{~K}$ 以上为第四段, 增 重量为 $4.4 \%$ 。实验结束时, 样品最终的总增重为 $123.0 \%$, 而 $\mathrm{MgB}_{2}$ 样品完全氧化后的理论增重为 $130.4 \%$ 。因此, 当加热至 $1665 \mathrm{~K}$ 时, $\mathrm{MgB}_{2}$ 样品已基 本完全氧化。

$\mathrm{MgB}_{2}$ 各阶段氧化产物的 SEM 照片和 XRD 图 谱分别如图 2 和图 3 所示, 化学分析结果如表 2 所示。

(1) $\mathrm{MgB}_{2}$ 氧化反应第一阶段

样品加热至 $1165 \mathrm{~K}$ 时, 处于 $\mathrm{MgB}_{2}$ 氧化的第一 阶段。氧化产物主要由 $\mathrm{MgO}$ 和未被氧化的 $\mathrm{MgB}_{2}$ 构成。化学分析结果显示, $\mathrm{MgO}$ 和 $\mathrm{B}_{2} \mathrm{O}_{3}$ 的摩尔比为 $1.14: 1$, 与 $\mathrm{MgB}_{2}$ 中镁元素和硼元素的摩尔比接近。 故推测此时发生了如下反应。

$$
\mathrm{MgB}_{2}+2 \mathrm{O}_{2}=\mathrm{MgO}+\mathrm{B}_{2} \mathrm{O}_{3}
$$

与此同时, $\mathrm{MgB}_{2}$ 样品中尚未发生氧化反应的硼 与镁的摩尔比约为 $2.06: 1$, 亦与图 3(a)中显示的信 息吻合。对比图 2(a b) 可知, 与未氧化前的微观形 貌相比, $\mathrm{MgB}_{2}$ 的晶体形状变得模糊, 且在其表面覆 盖了一层由 $\mathrm{MgO}$ 和 $\mathrm{B}_{2} \mathrm{O}_{3}$ 组成的疏松氧化层, 对反 应物扩散的阻碍作用较小, 氧化反应平稳进行, 表 现为 $\mathrm{TG}$ 曲线基本呈线性。 


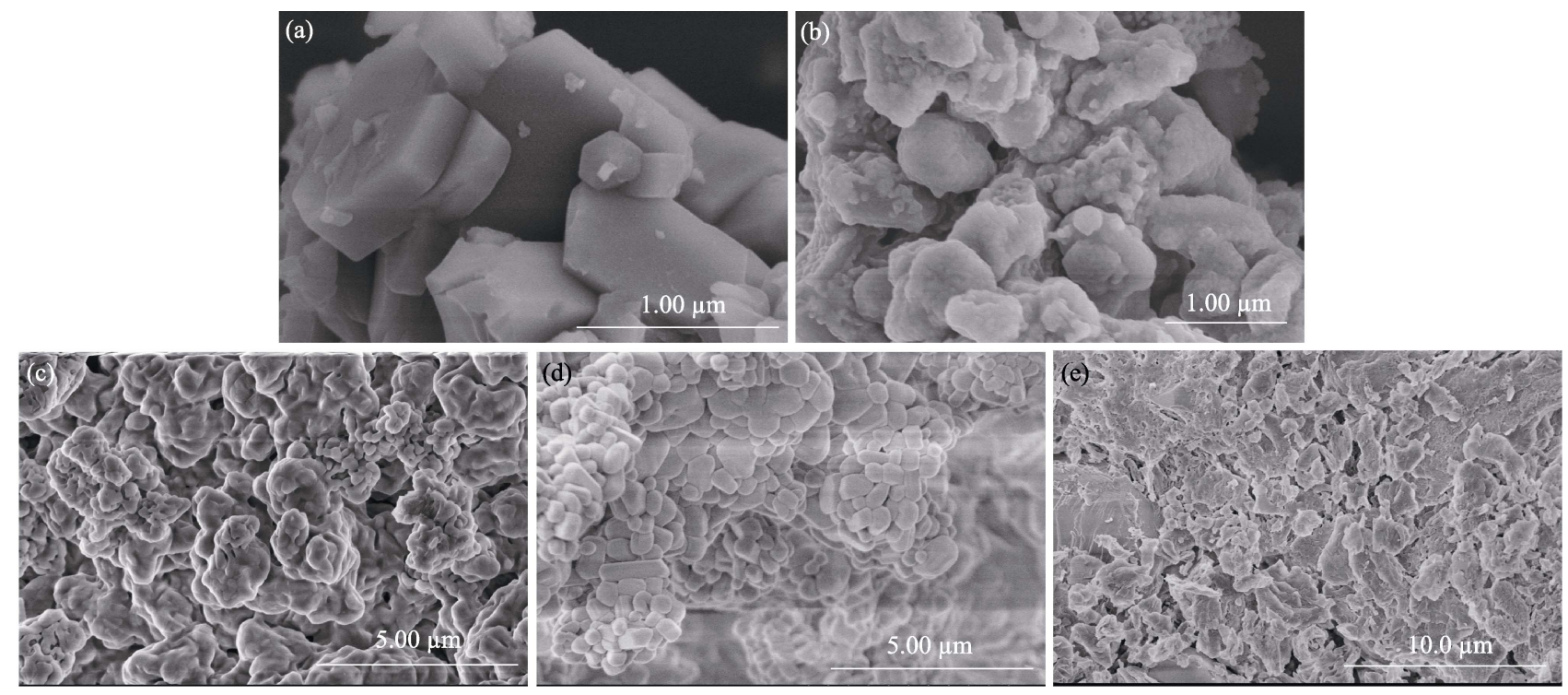

图 $2 \mathrm{MgB}_{2}$ 氧化产物的 SEM 照片

Fig. 2 SEM images of $\mathrm{MgB}_{2}$ oxidation residues

(a) Without oxidation; (b) $1165 \mathrm{~K}$; (c) $1300 \mathrm{~K}$; (d) $1405 \mathrm{~K}$; (e) $1520 \mathrm{~K}$

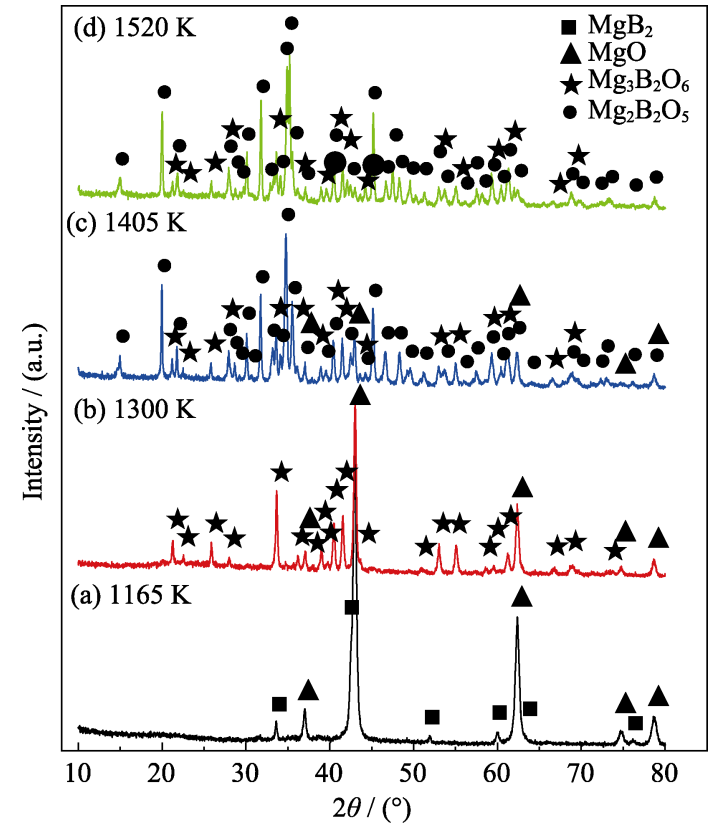

图 $3 \mathrm{MgB}_{2}$ 氧化产物的 XRD 图谱

Fig. 3 XRD patterns of $\mathrm{MgB}_{2}$ oxidation residues

\section{(2) $\mathrm{MgB}_{2}$ 氧化反应第二阶段}

由图 3(b) 可知, $1300 \mathrm{~K}$ 时 $\mathrm{MgB}_{2}$ 氧化产物中主要 物相为 $\mathrm{Mg}_{3} \mathrm{~B}_{2} \mathrm{O}_{6}$ 和 $\mathrm{MgO}$, 而 $\mathrm{MgB}_{2}$ 的衍射峰消失。 $\mathrm{MgB}_{2}$ 氧化产物中的 $\mathrm{MgO}$ 和 $\mathrm{B}_{2} \mathrm{O}_{3}$ 会进一步发生反 应，形成 $\mathrm{Mg}-\mathrm{B}-\mathrm{O}$ 三元氧化物。

$$
3 \mathrm{MgO}+\mathrm{B}_{2} \mathrm{O}_{3}=\mathrm{Mg}_{3} \mathrm{~B}_{2} \mathrm{O}_{6}
$$

$\mathrm{B}_{2} \mathrm{O}_{3}-\mathrm{MgO}$ 二元相图如图 $4^{[23]}$ 所示。由表 2 可 知, $1300 \mathrm{~K}$ 时 $\mathrm{MgO}$ 和 $\mathrm{B}_{2} \mathrm{O}_{3}$ 的摩尔比为 $3.04: 1$, 体 系状态处于图 4 中 $\mathrm{A}$ 点位置。在此条件下, 该体系 的稳态产物应为 $\mathrm{MgO}$ 和 $\mathrm{Mg}_{3} \mathrm{~B}_{2} \mathrm{O}_{6}$, 这与 XRD 图谱
表 $2 \mathrm{MgB}_{2}$ 热氧化产物的化学分析结果

Table 2 Chemical analytical results of $\mathrm{MgB}_{2}$ oxidation residues

\begin{tabular}{ccccr}
\hline $\begin{array}{c}\text { Temperature } \\
/ \mathrm{K}\end{array}$ & $n_{\mathrm{MgO}} / n_{\mathrm{B}_{2} \mathrm{O}_{3}}$ & $\begin{array}{c}\text { Fraction of } \\
\text { unoxidized } \\
\mathrm{Mg} / \mathrm{mol} \%\end{array}$ & $\begin{array}{c}\text { Fraction of } \\
\text { unoxidized } \\
\text { boron } / \mathrm{mol} \%\end{array}$ & $n_{\mathrm{B}} / n_{\mathrm{Mg}}$ \\
\hline 1165 & $1.14: 1$ & 81.11 & 83.38 & $2.06: 1$ \\
1300 & $3.04: 1$ & 22.73 & 74.62 & $6.56: 1$ \\
1405 & $1.52: 1$ & 6.35 & 38.35 & $12.08: 1$ \\
1520 & $1.26: 1$ & 2.14 & 22.30 & $20.89: 1$ \\
\hline
\end{tabular}

$n_{\mathrm{MgO}}$ : mole content of $\mathrm{MgO} ; n_{\mathrm{B}_{2} \mathrm{O}_{3}}$ : mole content $\mathrm{B}_{2} \mathrm{O}_{3} ; n_{\mathrm{B}}$ : mole content of the unoxidized boron; $n_{\mathrm{Mg}}$ : mole content of the unoxidized magnesium.

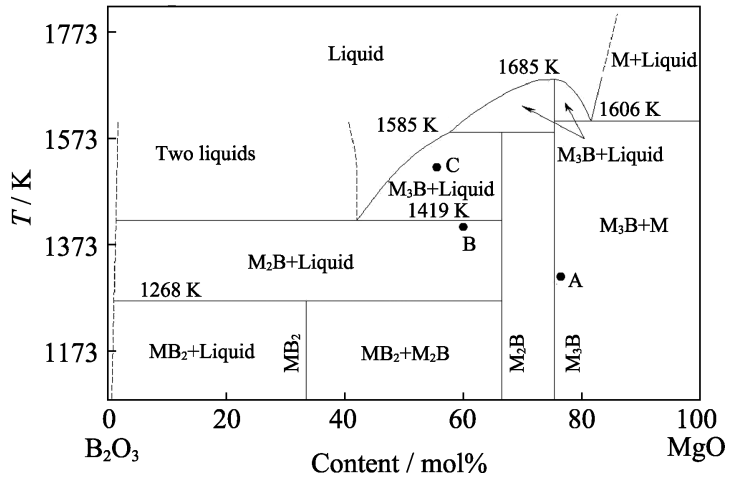

图 $4 \quad \mathrm{~B}_{2} \mathrm{O}_{3}-\mathrm{MgO}$ 二元相图 ${ }^{[23]}$

Fig. $4 \mathrm{~B}_{2} \mathrm{O}_{3}-\mathrm{MgO}$ binary phase diagram ${ }^{[23]}$

M: $\mathrm{MgO} ; \mathrm{MB}_{2}: \mathrm{MgB}_{4} \mathrm{O}_{7} ; \mathrm{M}_{2} \mathrm{~B}: \mathrm{Mg}_{2} \mathrm{~B}_{2} \mathrm{O}_{5} ; \mathrm{M}_{3} \mathrm{~B}: \mathrm{Mg}_{3} \mathrm{~B}_{2} \mathrm{O}_{6}$

所反映的产物信息相吻合。

此外, $1165 \mathrm{~K}$ 时 $\mathrm{MgB}_{2}$ 样品中未氧化的镁元素 的摩尔百分数为 $81.11 \%$, 而当温度升高至 $1300 \mathrm{~K}$ 后，这一比例仅为 $22.73 \%$, 降幅较大。与此同时, 
$\mathrm{MgB}_{2}$ 样品中未氧化的硼元素与镁元素的摩尔比为 6.56:1 (接近 $\mathrm{MgB}_{7}$ 分子的化学组成), 远高于第一 阶段的 2.06:1。这表明, 随着温度的升高, $\mathrm{MgB}_{2}$ 中 的镁元素会优先发生氧化反应。结合氧化产物的构 成，推测 $\mathrm{MgB}_{2}$ 氧化第二阶段中会发生如下反应:

$20 \mathrm{MgB}_{2}+17 \mathrm{O}_{2}=5 \mathrm{Mg}_{3} \mathrm{~B}_{2} \mathrm{O}_{6}+4 \mathrm{MgB}_{7}+\mathrm{MgO}+\mathrm{B}_{2} \mathrm{O}_{3}$

当温度升高至 $1405 \mathrm{~K}$ 时, 样品仍处于氧化过程 的第二阶段。如图 3(c)所示, $\mathrm{MgB}_{2}$ 样品的氧化产物 中除了 $\mathrm{MgO}$ 以外, 还包含 $\mathrm{Mg}_{2} \mathrm{~B}_{2} \mathrm{O}_{5} 、 \mathrm{Mg}_{3} \mathrm{~B}_{2} \mathrm{O}_{6}$ 两种 三元氧化物。且 $\mathrm{Mg}_{2} \mathrm{~B}_{2} \mathrm{O}_{5}$ 的 $\mathrm{X}$ 射线衍射峰强度最高, 为主要氧化产物。湿法化学分析结果显示, 氧化产 物中 $\mathrm{MgO}$ 和 $\mathrm{B}_{2} \mathrm{O}_{3}$ 的摩尔比由 3.04:1 降低至 $1.52: 1$ (如表 2 所示), 体系状态处于图 4 中 $\mathrm{B}$ 点位 置。在此条件下, 体系中稳定存在的物相应为 $\mathrm{Mg}_{2} \mathrm{~B}_{2} \mathrm{O}_{5}$, 这与图 3(c)所显示的信息吻合。同时, $\mathrm{MgB}_{2}$ 样品中未氧化硼元素与未氧化镁元素的摩尔 比由 $6.56: 1$ 增大至 $12.08: 1$, 硼元素的相对含量进 一步增大。此外, 如图 2(c d d) 所示, 此时 $\mathrm{MgB}_{2}$ 氧化 产物的颗粒间有明显空隙, 微观结构较疏松, 有利 于氧化反应的进行。

(3) $\mathrm{MgB}_{2}$ 氧化反应第三阶段

当加热至 $1520 \mathrm{~K}$ 时, 进入 $\mathrm{MgB}_{2}$ 氧化反应的第 三阶段, $\mathrm{MgB}_{2}$ 样品发生熔融现象, 冷却后形成质地 坚硬的硬块。如图 2(e)所示, 与上一阶段颗粒分明 的情况不同, 此时 $\mathrm{MgB}_{2}$ 样品的颗粒间呈现相互融 合的趋势。如图 3(d)所示, 与上一阶段相比, $\mathrm{MgB}_{2}$ 氧化产物 XRD 谱图中的 $\mathrm{MgO}$ 消失, 只剩下 $\mathrm{Mg}_{2} \mathrm{~B}_{2} \mathrm{O}_{5}$ 和 $\mathrm{Mg}_{3} \mathrm{~B}_{2} \mathrm{O}_{6}$ 两种三元氧化物。此时, $\mathrm{MgO}$ 和 $\mathrm{B}_{2} \mathrm{O}_{3}$ 的摩尔比为 $1.26: 1$ (详见表 2), 体系状态处 于图 4 中 $\mathrm{C}$ 点位置。在此条件下, 体系中稳定存在 的物相应为 $\mathrm{Mg}_{2} \mathrm{~B}_{2} \mathrm{O}_{5}$, 这与图 3(d)所显示的信息吻 合。此外, 样品中未氧化的硼元素和镁元素的摩尔 比由 $12.08: 1$ 进一步提高至 $20.89: 1$ 。

(4) $\mathrm{MgB}_{2}$ 氧化反应第四阶段

上一阶段的研究表明, $\mathrm{MgB}_{2}$ 在高温氧化条件下 会出现熔融现象, 无法从坩埚中取样分析。为进一 步研究 $\mathrm{MgB}_{2}$ 第四阶段的氧化反应, 本文利用粉体 挤压成型设备将 $\mathrm{MgB}_{2}$ 粉末压制成密实的立方体样 品, 并利用管式炉在空气环境下将其加热至 $1665 \mathrm{~K}$ 。 待样品冷却后, 利用扫描电镜(SEM)对块体的表层 结构进行观察分析, 其剖面形貌如图 5 所示。图 5 中左边为 $\mathrm{MgB}_{2}$ 块体内侧, 右边为外侧, 可见 $\mathrm{MgB}_{2}$ 块体的表面氧化层出现分层现象。外侧结构致密, 而内侧结构疏松多孔。在图 5 中横线指示的位置进 行 $\mathrm{X}$ 射线能谱线扫描分析, 结果如图 6 和表 3 所示。 如图 6 所示, 硼元素的含量在分层位置呈现出

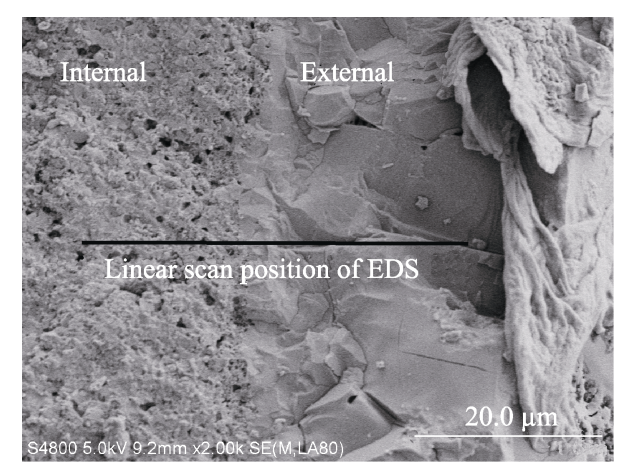

图 $5 \mathrm{MgB}_{2}$ 块体在 $1665 \mathrm{~K}$ 氧化后的表层剖面 SEM 照片 Fig. 5 Cross-sectional SEM image of oxide layer structure of $\mathrm{MgB}_{2}$ pellet after being heated up to $1665 \mathrm{~K}$

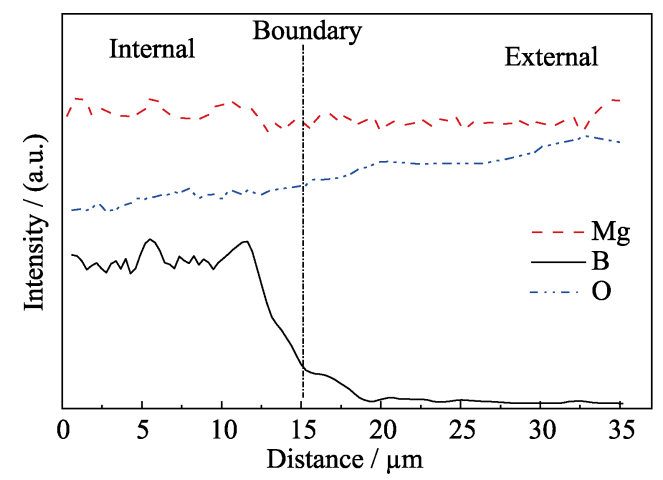

图 $6 \mathrm{MgB}_{2}$ 块体表层剖面的线扫描 $\mathrm{EDS}$ 能谱

Fig. 6 Linear EDS mapping of $\mathrm{MgB}_{2}$ pellet across the oxide layer

表 $3 \mathrm{MgB}_{2}$ 块体氧化表层的 $\mathrm{EDS}$ 能谱结果

Table 3 EDS results of the internal and external oxide layer of $\mathrm{MgB}_{2}$ pellet

\begin{tabular}{cccc}
\hline Position & $\mathrm{B} / \mathrm{mol} \%$ & $\mathrm{O} / \mathrm{mol} \%$ & $\mathrm{Mg} / \mathrm{mol} \%$ \\
\hline Internal & 11.78 & 51.22 & 37.00 \\
External & - & 56.43 & 43.57 \\
\hline
\end{tabular}

阶梯变化现象。结合表 3 中的数据可知, 外侧致密 结构主要由镁和氧两种元素组成, 即为 $\mathrm{MgO}$ 。打磨 掉块体表面的 $\mathrm{MgO}$ 层后, 对内侧氧化产物进行了 $\mathrm{XRD}$ 测试, 发现其主要成分为 $\mathrm{MgO} 、 \mathrm{Mg}_{2} \mathrm{~B}_{2} \mathrm{O}_{5}$ 及 $\mathrm{Mg}_{3} \mathrm{~B}_{2} \mathrm{O}_{6}$ (如图 7 所示)。此外, 在 $\mathrm{MgB}_{2}$ 块体周围的 坩埚表面沉积了一层透明状物质, 推测其为结晶后 的 $\mathrm{B}_{2} \mathrm{O}_{3}$ 。

由图 4 可知, 当温度超过 $1585 \mathrm{~K}$ 后, $\mathrm{Mg}_{2} \mathrm{~B}_{2} \mathrm{O}_{5}$ 会发生如下分解反应:

$$
\mathrm{Mg}_{2} \mathrm{~B}_{2} \mathrm{O}_{5}=\mathrm{Mg}_{3} \mathrm{~B}_{2} \mathrm{O}_{6}+\text { Liquid }
$$

文献研究成果表明, 液态 $\mathrm{B}_{2} \mathrm{O}_{3}$ 能显著降低固态 $\mathrm{MgO}$ 的熔点 ${ }^{[23]}$ 。反应(4)生成的液相即为 $\mathrm{MgO}(\mathrm{s})$ 溶 于 $\mathrm{B}_{2} \mathrm{O}_{3}(1)$ 后生成的溶液。故当加热至 $1665 \mathrm{~K}$ 后, $\mathrm{MgB}_{2}$ 块体的表面也会生成这种溶液。在此高温环境 下, 溶液中的 $\mathrm{B}_{2} \mathrm{O}_{3}$ 逐渐挥发, 而溶液中的 $\mathrm{MgO}$ 最 


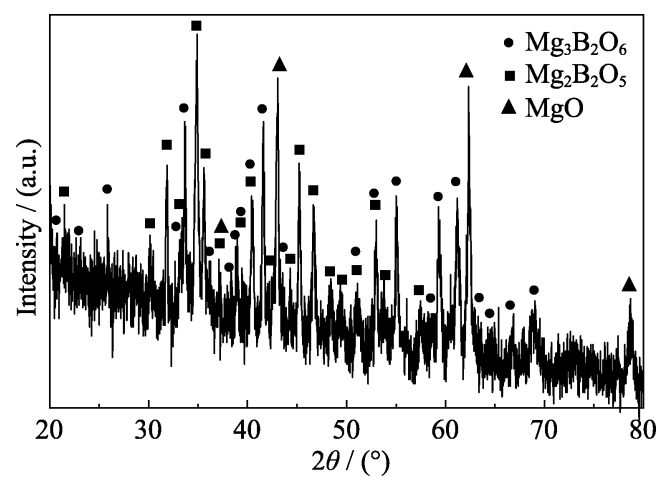

图 7 内层氧化物 XRD 图谱

Fig. 7 XRD pattern of the internal porous oxide layer

终结晶成致密结构。综合上述四个阶段的研究, 本 文揭示了 $\mathrm{MgB}_{2}$ 的氧化反应历程, 如图 8 所示。

\section{$2.3 \mathrm{MgB}_{2}$ 和无定型硼热氧化特性的对比}

张先瑞等 ${ }^{[24]}$ 采用超高温热重仪得到了无定形 硼粉在氧气环境下的氧化曲线。无定型硼样品纯度 为 $90 \%$, 热重仪升温速率为 $10 \mathrm{~K} \cdot \mathrm{min}^{-1}$, 温度范围 为室温至 $1973 \mathrm{~K}$, 实验结果如图 9 所示。

如图 9 所示, 在实验温度范围内, 根据样品增 重情况可将无定形嗍粉的热氧化曲线分为两段进行 分析: (1)第一阶段增重出现在 $1037 \mathrm{~K}$ 。当第一阶段 反应结束时 (温度约为 $1270 \mathrm{~K}$ ), 样品的氧化百分数 约为 $38.2 \%$ 。(2)第二阶段的反应主要发生在 $1919 \mathrm{~K}$

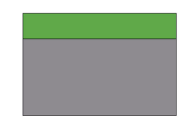

Stage A

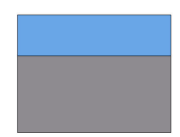

Stage B

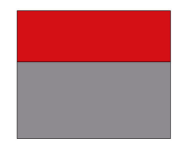

Stage C

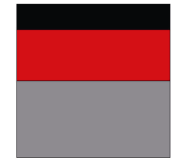

Stage D
Porous $\mathrm{MgO}+\mathrm{B}_{2} \mathrm{O}_{3}$

$\mathrm{MgO}+\mathrm{Mg}_{2} \mathrm{~B}_{2} \mathrm{O}_{5}+\mathrm{Mg}_{3} \mathrm{~B}_{2} \mathrm{O}_{6}$

$\mathrm{Mg}_{3} \mathrm{OB}_{2} \mathrm{O}_{6}+\mathrm{Mg}_{2} \mathrm{~B}_{2} \mathrm{O}_{5}$

Condensed $\mathrm{MgO}$

图 $8 \mathrm{MgB}_{2}$ 的氧化反应历程

Fig. 8 Schematic diagram of evolution of $\mathrm{MgB}_{2}$ oxidation reaction

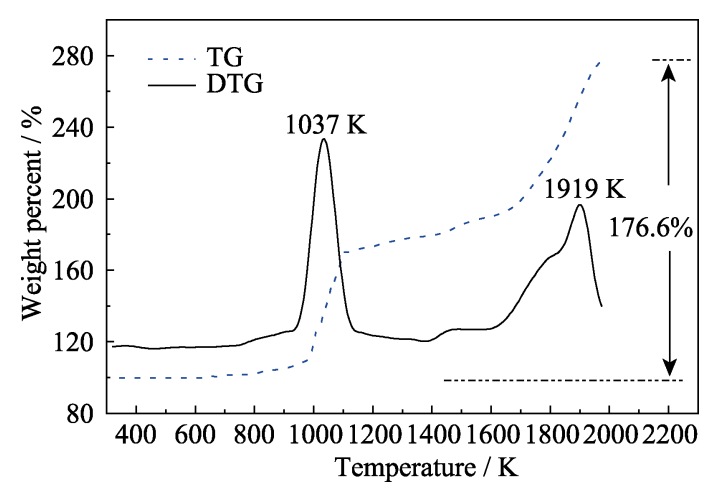

图 9 超高温条件下无定形嗍的热氧化 TG 和 DTG 曲线 ${ }^{[24]}$

Fig. 9 TG and DTA curves of amorphous boron oxidation under ultra-high temperature ${ }^{[24]}$

附近。在 $1973 \mathrm{~K}$ 实验结束时, 无定型硼粉的氧化百 分数为 $88.4 \%$ 。可知, 无定型硼粉在氧化反应第二 阶段的增重远大于在第一阶段的增重, 即其主要的 氧化增重发生在 $1919 \mathrm{~K}$ 附近。

对比可知, $\mathrm{MgB}_{2}$ 的氧化反应主要发生在 $873 \mathrm{~K}$ 至 $1480 \mathrm{~K}$, 该温度区间远低于无定形硼的主要氧化 峰温度 $(1919 \mathrm{~K})$ 。当环境温度为 $1665 \mathrm{~K}$ 时, $\mathrm{MgB}_{2}$ 的 氧化百分数为 $94.3 \%$, 而无定形硼仅为 $43.6 \%$ 。

综合分析两者的热氧化历程, 无定型嗍在 $1037 \mathrm{~K}$ 发生第一阶段氧化反应后, 其表面即覆盖了 $\mathrm{B}_{2} \mathrm{O}_{3}$ 氧化层。由于 $\mathrm{B}_{2} \mathrm{O}_{3}$ 的熔点仅为 $723 \mathrm{~K}$, 沸点更 是高达 $2320 \mathrm{~K}$, 导致该氧化层表现为粘度较高的液 态氧化层, 紧密地包裹在了嗍颗粒表面, 阻碍了后 续氧化反应。只有在 $1700 \mathrm{~K}$ 以上的高温条件下才能 加速挥发, 发生无定型硼的第二阶段氧化。而 $\mathrm{MgB}_{2}$ 的氧化过程则不同, 反应产生的 $\mathrm{MgO}$ 与液态 $\mathrm{B}_{2} \mathrm{O}_{3}$ 接触紧密, 并进一步反应生成 $\mathrm{Mg}_{2} \mathrm{~B}_{2} \mathrm{O}_{5}$ 和 $\mathrm{Mg}_{3} \mathrm{~B}_{2} \mathrm{O}_{6}$ 等三元氧化物。参照 $\mathrm{B}_{2} \mathrm{O}_{3}-\mathrm{MgO}$ 相图可知, 这些三 元氧化物的熔点相较于 $\mathrm{B}_{2} \mathrm{O}_{3}$ 显著提升, 在氧化反应 历程中主要呈固态, 避免了颗粒表面液态 $\mathrm{B}_{2} \mathrm{O}_{3}$ 氧化 层的聚集, 颗粒内部与氧化性气氛的接触更为顺畅, 使得 $\mathrm{MgB}_{2}$ 相较于无定型硼而言能够在较低的温度 下充分氧化放热。

\section{3 结论}

1) $\mathrm{MgB}_{2}$ 的燃烧热值略高于无定型硼, 但燃烧 效率(62\%)却明显高于无定型硼(38\%), 具备良好的 应用基础。

2) 在氧化反应过程中, $\mathrm{MgB}_{2}$ 表面会生成 $\mathrm{Mg}_{-}$ B-O 三元氧化物, 这些三元氧化物的熔点相较于 $\mathrm{B}_{2} \mathrm{O}_{3}$ 显著提升, 在氧化反应历程中主要呈固态, 避 免了颗粒表面液态 $\mathrm{B}_{2} \mathrm{O}_{3}$ 氧化层的聚集，这一特性有 利于其快速氧化放热。与无定形嗍相比, $\mathrm{MgB}_{2}$ 可在 较低的温度下实现较高的氧化百分数, 热氧化特性 更优。

\section{参考文献:}

[1] MITSUNO M, KUWAHARA T, KOSAKA K, et al. Combustion of Metallized Propellants for Ducted Rockets. AIAA-87-1724, 1987.

[2] MIYAYAMA T, OSHIMA H, TOSHIYUKI S, et al. Improving Combustion of Boron Particles in Secondary Combustor of Ducted Rockets. AIAA 2006-5250, 2006.

[3] OBUCHI K, TANABE M, KUWAHARA T. Ignition Characteristics of Boron Particles in the Secondary Combustor of Ducted Rockets-Effects of Magnalium Particle Addition. AIAA 2008-943, 2008. 
[4] LIU T K, LUH S P, PERNG H C. Effect of boron particles surface coating on combustion of solid propellants for ducted rockets. Propellants, Explosives, Pyrotechnics, 1991, 16(4): 156-166.

[5] BOYD D D, CHILDS L B. Method of Coating Boron Particles with Ammonium Perchlorate. USP 3976521, 1976-8-24.

[6] LI S F, JIN R. Improvement of Combustion Characteristics of Solid Propellant with Coated Boron. AIAA 99-2633, 1999.

[7] SHYU I M, LIU T K. Combustion characteristics of gap-coated boron particles and the fuel-rich solid propellant. Combustion and Flame, 1995, 100(4): 634-644.

[8] YEH C L. Ignition and Combustion of Boron Particles. Pennsylvania: The Pennsylvania State University, 1995.

[9] YEH C L, KUO K K. Ignition and combustion of boron particles. Progress in Energy and Combustion Science, 1996, 22(6): 511-541.

[10] BESSER H L, STRECKER R. Overview of Boron Ducted Rocket Development during the Last Two Decades. Combustion of BoronBased Solid Propellants and Solid Fuels. Boca Raton: Begell House Publishing Co. and CRC Press, 1993: 133-178.

[11] LIU T K, SHYU I M, HSIA Y S. Effect of fluorinated graphite on combustion of boron and boron-based fuel-rich propellants. Journal of Propulsion and Power, 1996, 12(1): 26-33.

[12] MACRI B J. Process for Making Spheroidal Agglomerates. USP 3646174, 1972-2-29.

[13] MACRI B J. Boron-Fuel-Rich Propellant Compositions. USP 3986909, 1976-10-19.

[14] HSIEH W H, PERETZ A, HUANG I T, et al. Combustion behavior of boron-based BAMO/NMMO fuel-rich solid propellants. Journal of Propulsion and Power, 1991, 7(4): 497-504.

[15] YANG A S, HUANG I T, HSIEH W H, et al. Burning-Rate Characteristics of Boron/[BAMO/NMMO] Fuel-Rich Solid Propellant
Under Broad Ranges of Pressure and Temperature. Combustion of Boron-based Solid Propellants and Solid Fuels. Boca Raton: Begell House Publishing Co. and CRC Press, 1993: 412-426.

[16] HSIA H T S. Air-Augmented Combustion of Boron and BoronMetal Alloys. AFRPL-TR-71-80, 1971.

[17] MESTWERDT R, SELZER H. The Combustion of a BoronLithium Compound with Respect to Air Augmented Rockets. AIAA 75-247, 1975.

[18] MESTWERDT R, SELZER H. Experimental investigation of boron/ lithium combustion. AIAA Journal, 1976, 14(1): 100-102.

[19] MOTA J M, MARTINEZ M A, VELASCO F J, et al. A method of making boride and vitreous compound by powder metallurgy. Journal of Materials Processing Technology, 2003, 143-144: 764768.

[20] MOTA J M, ABENOJAR J, MARTINEZ M A, et al. Borides and vitreous compounds sintered as high-energy fuels. Journal of Solid State Chemistry, 2004, 117(2): 619-627.

[21] MOTA J M, MARTINEZ M A, VELASCO F, et al. Preparation of aluminium boride by powder technology. Ceramics International, 2004, 30(2): 301-306.

[22] Ascharite Ores-determination of Boric Anhydride Content-Volumetric Method. HG/T 2956. 3-2001, 2001.

[23] MUTLUER T, TIMUCIN M. Phase equilibria in the system $\mathrm{MgO}-\mathrm{B}_{2} \mathrm{O}_{3}$. Journal of the American Ceramic Society, 1975, 58(5/6): 196-197.

[24] ZHANG XIAN-RUI, WANG YUAN-YUAN, CHEN TAO, et al. Thermal Oxidation Characteristics of Amorphous Boron Powder. The $29^{\text {th }}$ Academic Annual Conference of Solid Propulsion Committee of Chinese Society of Astronautics, Ningbo, 2012: $611-614$. 